\section{Um estudo sobre a validade de construto da Parent-Child Conflict Tactics Scale (CTSPC) em uma amostra populacional urbana do Nordeste brasileiro}

\author{
A study on the construct validity of the \\ Parent-Child Conflict Tactics Scale (CTSPC) \\ in an urban population in Northeast Brazil
}

\author{
1 Instituto de Saúde Coletiva, \\ Universidade Federal da \\ Bahia, Salvador, Brasil. \\ 2 Instituto de Psicologia, \\ Universidade Federal da \\ Bahia, Salvador, Brasil. \\ 3 Instituto de Medicina \\ Social, Universidade do \\ Estado do Rio de Janeiro, \\ Rio de Janeiro, Brasil. \\ Correspondência \\ C. B. Bonfim \\ Instituto de Saúde Coletiva \\ Universidade Federal da \\ Bahia. \\ Rua Basílio da Gama s/n, \\ Campus Universitário Canela, \\ Salvador, BA 40110-040, \\ Brasil. \\ bonfimcamila@yahoo.com.br
}

\begin{abstract}
The Parent-Child Conflict Tactics Scale (CTSPC) is one of the most widely used instruments in the world for investigating domestic violence against children, but targeted use has proven inadequate given the phenomenon's complexity. This study focused on the factor structure of CTSPC scales in an urban population in Northeast Brazil. We conducted a cross-sectional study in a cohort of 1,370 children in Salvador, Bahia State. Factor analysis with promax oblique rotation was performed, and the Kuder-Richardson coefficient was calculated. Factor analysis showed a different distribution of items in the factors as compared to the original instrument. Violence showed a gradual profile in each factor. The Kuder-Richardson coefficient was 0.63 for factor 1, 0.59 for factor 2 , and 0.42 for factor 3 . The items behaved differently from the original instrument, corroborating international studies. These findings support proposing a resizing of the CTSPC.
\end{abstract}

Child Abuse; Domestic Violence; Urban Population; Statistical Factor Analysis
Camila Barreto Bonfim 1

Darci Neves Santos 1

Igor Gomes Menezes 2

Michael Eduardo Reichenheim 3

Mauricio Lima Barreto 1

\section{Introdução}

A violência doméstica contra a criança vem sendo cada vez mais estudada na realidade brasileira, revelando-se um problema de saúde pública. Estudo internacional sobre aprovação familiar do uso de punição corporal contra a criança revelou que, dos 33 países investigados, o Brasil ocupa a 16a posição no ranque ${ }^{1}$. Pesquisa epidemiológica recente em serviços de emergência em diversas capitais do Brasil identificou que, entre os anos de 2006 e 2007, houve 10.610 ocorrências devido a violências, sendo que 519 (5\%) referiam-se a crianças abaixo de 10 anos 2 . A violência física é a mais frequente $(42,2 \%)$, seguida da violência psicológica com $9,4 \%$, sendo a residência o local onde mais ocorrem tais fenômenos $(55,1 \%)$. Esses dados são, muitas vezes, subnotificados, ainda que existam políticas de proteção da criança que incentivem a notificação dos casos, tais como o Estatuto da Criança e do Adolescente ${ }^{3}$, a Política Nacional de Redução da Morbimortalidade por Acidentes e Violências 4 e estratégias de vigilância como o Sistema de Vigilância de Violências e Acidentes 5, proposto pelo Ministério da Saúde. Encontram-se ainda muitas dificuldades para estimar a magnitude desse fenômeno, devido a diferentes definições do termo e diversidade de fontes de informações sobre o tema 6 .

A complexidade inerente a tais fenômenos exige a elaboração de instrumentos que mensu- 
rem os construtos de maneira acurada. Tais instrumentos procuram medir atributos por meio de itens que possuem diferentes grandezas, representados por escores numéricos 7 . Cada variável expressa um traço não diretamente observado, como é o caso da percepção do indivíduo sobre o uso de violência física ou psicológica contra a criança. Não sendo possível mensurar objetivamente esses traços, utilizam-se perguntas estruturadas para avaliar um construto específico. Nesse sentido, é fundamental que esses instrumentos representem a percepção que os sujeitos têm sobre os fenômenos estudados.

Nos estudos sobre violência, verificou-se que, além de não ser um fenômeno facilmente mensurável objetivamente, também o é de difícil acesso pelo preconceito e estigma envolvendo os vitimizados e o medo de represálias pelas informações prestadas ${ }^{8}$. Instrumentos que mensuram esse construto de forma direta usando perguntas como "Você sofreu violência sexual?" mostraram-se pouco sensíveis e confiáveis 9 . Pesquisas sobre a acurácia desses instrumentos identificaram que a sensibilidade aumentava quando os indivíduos respondiam a itens ou perguntas múltiplas descrevendo experiências de maus-tratos que utilizavam termos objetivos e estritamente comportamentais ${ }^{9}$.

Ainda que o tema tenha alcançado relevância na literatura, são escassos os instrumentos validados e padronizados para o contexto brasileiro. Estudo epidemiológico, realizado em Ribeirão Preto (São Paulo), utilizou a Cartilha Epidemiológica, instrumento argentino apenas traduzido e adequado semanticamente para o Brasil 10,11, para avaliar a suspeição de maus-tratos em escolares de até 10 anos, nos últimos 12 meses, descrevendo diversas tipologias de violência doméstica contra a criança, conforme observação do professor. Uma revisão de literatura recente apontou apenas três instrumentos traduzidos e adaptados para a realidade brasileira, avaliando a violência doméstica contra a criança pelo relato da própria família ${ }^{8}$. O primeiro deles, o Questionário de Trauma na Infância 12,13 , é preferencialmente utilizado com população adolescente a partir de 12 anos e avalia experiências pregressas que incluem abusos físico, emocional e sexual, negligência física e negligência emocional. $\mathrm{O}$ segundo é o WorldSAFE Core Questionnaire on Domestic Violence 14,15, desenvolvido pelo grupo World Studies of Abuse in Family Environments, projeto multinacional que tem investigado a violência doméstica em países como o Brasil, Chile, Egito, Índia, Filipinas e Estados Unidos. $\mathrm{O}$ último concerne à Parent-Child Conflict Tactics Scale (CTSPC) 16,17, instrumento escolhido para esta pesquisa pelo seu uso internacional
18,19,20,21,22,23,24,25, tendo sido traduzido, validado e adaptado em diversos países 26 , além de ter servido de base para a construção de questionário elaborado pelo WorldSAFE. Há ainda outros instrumentos publicados que avaliam esse fenômeno, porém na perspectiva do adulto que sofreu de violência familiar em sua própria infância 27 , não sendo esse o foco do estudo.

\section{A CTSPC e suas evidências de validade de construto}

A CTSPC é uma escala desenvolvida pelo Family Violence Research Program 17, nos Estados Unidos, composta originalmente por 22 itens que investigam a violência doméstica a partir do comportamento parental de educar filhos (Tabela 1). Três dimensões (subescalas) são avaliadas pela CTSPC: disciplina não-violenta (4 itens), agressão psicológica (5 itens) e violência física (13 itens), sendo essa última subdividida conforme a gravidade em punição corporal (6 itens), maus-tratos físicos (3 itens) e maus-tratos físicos graves (4 itens).

No Brasil, a CTSPC foi adaptada transculturalmente e validada em estudo com 774 mães, entrevistadas em três maternidades públicas, no Rio de Janeiro, nas primeiras 48 horas de puerpério ${ }^{28}$. No processo, avaliou-se a equivalência conceitual e de itens, bem como a equivalência semântica com o objetivo de atestar a validade de conteúdo da medida. Os resultados revelaram que os 22 itens do instrumento estavam adequados à cultura brasileira e tinham seu uso recomendado para esse contexto. No entanto, a análise fatorial exploratória identificou problemas em alguns itens, tais como a baixa carga fatorial de um item que supostamente deveria pertencer a uma determinada escala 28 . Para manter a comparabilidade com estudos internacionais, optouse por não retirar os itens problemáticos.

A análise fatorial exploratória (AFE) no estudo de validação de constructo realizado no Rio de Janeiro revelou ainda que o item F ("Você falou alto, berrou ou gritou com a criança?”), da escala de agressão psicológica, apresentou maior carga no fator correspondente à escala de punição corporal 12. O item V ("Você deu um tapa/bofetada no rosto, na cabeça ou nas orelhas da criança?"), da escala de maus-tratos físicos, apresentou maior carga no fator de agressão psicológica. Já o item R ("Você deu beliscão na criança?”), da escala de punição corporal, obteve maior carga fatorial na dimensão de maus-tratos físicos. Esse argumento tem sido corroborado também por pesquisas internacionais 21 . Nos Estados Unidos, foi realizado estudo longitudinal 
Itens da Parent-Child Conflit Tactics Scale (CTSPC), versão adaptada para o Brasil.

\begin{tabular}{|c|c|c|}
\hline Item & Dimensão & Pergunta \\
\hline A & DNV & Você explicou à criança por que o que ele(a) estava fazendo estava errado? \\
\hline B & DNV & $\begin{array}{l}\text { Você o(a) colocou de castigo do tipo: mandou-o(a) ficar em seu quarto ou em qualquer outro } \\
\text { lugar? }\end{array}$ \\
\hline C & PC & Você sacudiu ( $x x x$ - nome da criança)? \\
\hline $\mathrm{D}$ & PC & $\begin{array}{l}\text { Você bateu no bumbum dele(a) com alguma coisa como um cinto, chinelo, escova de cabelo, } \\
\text { vara ou outro objeto duro? }\end{array}$ \\
\hline E & DNV & Você deu a ele(a) outra coisa para fazer em vez daquilo que ele(a) estava fazendo de errado? \\
\hline $\mathrm{F}$ & AP & Você falou alto, berrou ou gritou com ( $x x x$ - nome da criança)? \\
\hline G & MF & Você bateu com a mão fechada ou deu um chute com força nele(a)? \\
\hline $\mathrm{H}$ & PC & Você deu uma palmada no bumbum de ( $x x x$ - nome da criança)? \\
\hline I & MFG & Você o(a) agarrou pelo pescoço e o(a) sacudiu? \\
\hline $\mathrm{J}$ & AP & Você xingou ou praguejou, quer dizer, rogou praga, contra ele(a)? \\
\hline $\mathrm{K}$ & MFG & $\begin{array}{l}\text { Você bateu muito em ( } x x x \text { - nome da criança), ou seja, bateu nele(a) sem parar, o máximo que V/S } \\
\text { conseguiu? }\end{array}$ \\
\hline L & AP & Você disse alguma vez que iria expulsá-lo(a) de casa ou enxotá-lo(a) para fora de casa? \\
\hline M & MFG & Você queimou ( $x x x$ - nome da criança) ou derramou líquido quente nele(a) de propósito? \\
\hline $\mathrm{N}$ & $\mathrm{AP}$ & Você ameaçou dar um tapa nele(a), mas não deu? \\
\hline $\mathrm{O}$ & MF & $\begin{array}{l}\text { Você bateu em alguma parte do corpo dele(a) diferente do bumbum com alguma coisa como um } \\
\text { cinto, chinelo, escova de cabelo, vara ou outro objeto duro? }\end{array}$ \\
\hline $\mathrm{P}$ & PC & Você deu um tapa na mão, no braço ou na perna de ( $x x x$ - nome da criança)? \\
\hline Q & DNV & Você tirou as regalias dele(a) ou o(a) deixou sem sair de casa? \\
\hline $\mathrm{R}$ & PC & Você deu beliscão em ( $x x x$ - nome da criança)? \\
\hline $\mathrm{S}$ & MFG & Você o(a) ameaçou com uma faca ou arma? \\
\hline $\mathrm{T}$ & MF & Você jogou (xxx - nome da criança) no chão? \\
\hline$U$ & AP & Você o(a) chamou de estúpido(a), burro(a), preguiçoso(a) ou de outra coisa parecida? \\
\hline $\mathrm{V}$ & MF & Você deu um tapa/bofetada no rosto, na cabeça ou nas orelhas de ( $x x x$ - nome da criança)? \\
\hline
\end{tabular}

com 630 famílias havaianas, o qual identificou correlações altas entre cargas fatoriais de itens que não faziam parte das escalas originais 20 . A AFE revelou cinco fatores, agrupando itens das escalas de punição corporal, maus-tratos físicos e agressão psicológica, os quais foram denominados: punição corporal e verbal comum; ataque à autoestima da criança; bater na criança com um objeto; negligência. Esse último fator não pode ser comparado com a versão brasileira, tendo em vista que não foi adaptado para esse contexto.

Outro estudo norte-americano com 595 famílias do Havaí realizou AFE a partir dos fatores acima descritos, e, novamente, observou-se que alguns itens tiveram cargas fatoriais mais elevadas em escalas às quais não pertenciam originalmente. Também observaram que o item V ("Você deu um tapa/bofetada no rosto, na cabeça ou nas orelhas da criança?") apresentou maior carga no fator de agressão psicológica, achado similar ao estudo de validação brasileiro. Verificaram-se ainda diferenças no agrupamento de itens através da AFE em estudo realizado em Quebec com 2.465 famílias 25. O Fator 1 englobou itens que envolviam aprovação do uso da violência para disciplinamento, e o Fator 2 incluiu aqueles itens referentes à reprovação do uso da violência.

Apresentamos um panorama dos estudos que examinaram a estrutura fatorial e a consistência interna de várias aplicações da escala em relação ao estudo original (Tabela 2). A maioria das investigações ocorreu nos últimos 12 anos, com participação de famílias americanas, utilização da AFE, com rotação varimax, tendo encontrado quatro fatores. A AFE é o tipo de análise mais adequada quando não há conhecimento acumulado sobre o instrumento avaliado ${ }^{29}$. No entanto, para propor mudanças estruturais no instrumento, seria necessária a realização de 
Análise fatorial e consistência interna da Parent-Child Conflit Tactics Scale (CTSPC) em estudos nacionais e internacionais.

\begin{tabular}{|c|c|c|c|c|c|c|}
\hline Referência & Ano & $\begin{array}{c}\text { População } \\
\text { (famílias) }\end{array}$ & $\begin{array}{l}\text { Tipo de } \\
\text { análise } \\
\text { fatorial }\end{array}$ & $\begin{array}{c}\text { Número de } \\
\text { fatores }\end{array}$ & $\begin{array}{l}\text { Tipo de } \\
\text { rotação }\end{array}$ & $\begin{array}{l}\text { Variação da } \\
\text { consistência } \\
\text { interna }(\alpha)\end{array}$ \\
\hline Straus et al. 17 & 1998 & 1.000 americanos & AFE & 4 & Varimax & $0,55-0,70$ \\
\hline Dietz 24 & 2000 & 1.000 americanos & - & - & - & $0,25-0,54$ \\
\hline Reichenheim \& Moraes 28 & 2006 & 774 brasileiros & AFE & 4 & Varimax & $0,48-0,66$ \\
\hline Duggan et al. 20 & 2004 & 630 americanos & AFE & 4 & Varimax & - \\
\hline Windham et al. 21 & 2004 & 595 americanos & AFE & 4 & Varimax & - \\
\hline Clement \& Bouchard 25 & 2005 & 2.465 canadenses & AFE & 2 & Varimax & $0,60-0,66$ \\
\hline Downs et al. 23 & 2006 & 447 americanos & - & - & - & 0,59 \\
\hline Lee et al. 22 & 2008 & 1.257 americanos & - & - & - & $0,50-0,60$ \\
\hline Kelleher et al. 30 & 2008 & 3.000 americanos & - & - & - & $0,39-0,77$ \\
\hline
\end{tabular}

AFE: análise fatorial exploratória; $\alpha$ : alfa de Cronbach.

uma análise fatorial confirmatória (AFC), o que esses estudos não propõem, apesar de promoverem mudanças no agrupamento dos itens. Além disso, a rotação varimax não seria a mais adequada, visto que não pressupõe correlação entre os fatores, o que não parece aplicável à CTSPC.

Com relação à consistência interna, calculada pelo alfa de Cronbach, os estudos identificaram uma variação entre 0,25 e 0,77 (Tabela 2). As menores correlações foram encontradas nas escalas que avaliaram os maus-tratos físicos 17,24,30, o que foi explicado pelos autores devido à raridade do evento. Poucas respostas afirmativas foram encontradas nessa subescala, impactando no valor do alfa. As demais escalas tiveram uma consistência interna considerada aceitável, demonstrando que há uma homogeneidade dos itens na avaliação da violência doméstica contra a criança. Também não há itens redundantes, tendo em vista que as correlações são inferiores a 0,95 29. Todos os estudos calcularam a consistência interna por meio do alfa de Cronbach, índice mais adequado para avaliar variáveis quantitativas, o que não é o caso desse instrumento. O Kuder-Richardson seria um índice mais adequado para essas análises, pois avalia a consistência interna de variáveis de natureza qualitativa 29.

Apesar desses limites, essas evidências corroboram o que a literatura revela: coocorrência e relação entre esses tipos de violência, além de uma impossibilidade de compreensão do fenômeno de forma segmentada 25. Geralmente, a agressão psicológica vem acompanhada de punições corporais e vice-versa 31 . Steinmetz 31 propôs uma tipologia do padrão familiar no uso da violência doméstica contra a criança de acordo com o uso da agressão psicológica e violência física ou ambas as estratégias na resolução de conflitos: pacificistas que utilizariam baixos níveis de agressão psicológica e violência física; ameaçadores que utilizariam altos níveis de agressão verbal e baixos níveis de violência física; agressores silenciosos os quais usariam baixos níveis de agressão psicológica e altos níveis de violência física; espancadores gritantes que utilizariam altos níveis de agressão psicológica e violência física. Ainda que essa proposta não tenha sido testada empiricamente por meio de análise fatorial, percebe-se que se coaduna com as evidências trazidas pelos estudos, os quais identificaram correlações entre diferentes tipos de violência a partir de análises fatoriais (Tabela 2). A violência doméstica contra a criança é um fenômeno multifacetado e não pode ser estudado de forma segmentada, conforme originalmente proposto por Straus et al. 17 .

Este artigo tem como objetivo estudar a validade de construto da CTSPC por meio da AFC e da consistência interna, em uma amostra populacional urbana do Nordeste brasileiro e compará-la aos resultados do estudo original de Straus et al. 17, do estudo de validação brasileiro e em demais pesquisas que tenham publicado resultados sobre a validade de construto da CTSPC no contexto internacional. 


\section{Método}

\section{Desenho de estudo, população e área}

Trata-se de um estudo transversal integrado ao programa Social Changes, Asthma and Allergy in Latin America (SCAALA), conduzido na cidade de Salvador, Bahia, Brasil em 2006 32. O SCAALA é um programa composto por atividades de pesquisa realizadas no Brasil e no Equador. No Brasil, o principal objetivo deste estudo foi investigar a associação entre prevalência de asma e outras doenças alérgicas e potenciais fatores de risco, como exposições a infecções na infância, bem como fatores ambientais, nutricionais, imunológicos e psicossociais.

A população do estudo foi selecionada por meio de amostragem aleatória e recrutada a partir de 24 microáreas geográficas representativas da cidade de Salvador que recentemente haviam passado por tratamento sanitário de bacias de esgotamento 32 .

A amostra deste estudo foi composta de 1.370 crianças, sendo 731 meninos $(53,4 \%)$ e 639 meninas $(46,6 \%)$. A idade média foi de 7,1 anos (SD = 1,7), com mínimo de quatro e máximo de sete anos. Já a idade média das mães foi de 32,1 (SD = $6,7)$, variando entre 18 e 56 anos. A maioria das genitoras estudou de 5-8 anos (48\%). Os respectivos respondentes foram selecionados a partir de 1.445 sujeitos que faziam parte do projeto SCAALA. A taxa de não-resposta foi de 5,2\%.

\section{Coleta de dados}

Essa ocorreu por meio de visitas domiciliares realizadas por uma equipe treinada, constituída de psicólogos e estudantes de Psicologia. As informações foram respondidas, em sua maioria, pelas genitoras (75\%), e os demais respondentes eram avós, pais, irmãos ou vizinhos responsáveis pelo cuidado da criança.

Este estudo foi aprovado pelo Comitê de Ética em Pesquisa do Instituto de Saúde Coletiva da Universidade Federal da Bahia e seguiu os princípios éticos exigidos em pesquisa com seres humanos contidos na Declaração de Helsinki da Associação Médica Mundial.

\section{Análise de dados}

Inicialmente, a fim de verificar qual estrutura fatorial responde melhor pela variância explicada do construto, foi realizada uma AFE pelo método de fatores principais, e realizou-se rotação oblíqua do tipo promax, a qual assume que os fatores estão correlaciona-dos 29 . Tendo em vista que os itens são dicotômicos, aplicou-se a matriz de correlação tetracórica ${ }^{33}$. Foram considerados três critérios para se inferir um item como inadequado a partir dos resultados da análise fatorial: (1) apresentar valor absoluto da carga fatorial principal do item menor do que 0,30; (2) possuir cargas fatoriais similares em dois ou mais fatores em um mesmo item, com a diferença entre valores absolutos das cargas fatoriais dos itens menor do que 0,10; e (3) não similaridade entre o item e o construto teórico expresso pelo fator 34,35,36. Para identificar o número de fatores, foram utilizados o critério de eigenvalue acima de 1 , o ponto de inflexão do scree-plot e a disposição dos fatores, considerando a saturação item-fator 34 .

Para verificar a adequação do modelo encontrado a partir da AFE, realizou-se uma AFC através do MPlus 6.1 (Muthén \& Muthén, Los Angeles, Estados Unidos). Esse procedimento foi realizado previamente em outros estudos 37 , sendo apropriado para testar a adequação do modelo. A AFC consiste em uma técnica de análise multivariada, inserida nos modelos de equações estruturais, a qual tem sido bastante utilizada na Psicologia 38,39,40, porém ainda pouca aplicada em Epidemiologia 41. Sua vantagem consiste em testar a aplicabilidade de um modelo teórico baseado em variáveis latentes (construtos teóricos) por meio de variáveis observáveis (os itens) ${ }^{41}$. Para avaliar a adequação do modelo, foram utilizadas algumas medidas de ajustamento: Comparative Fit Index (CFI), Tucker-Lewis Index (TFI) e Root Mean Square Error of Aproximation (RMSEA). Consideraram-se, como adequados, os modelos que apresentaram CFI e TFI maiores do que 0,90 e RMSEA inferior a 0,0542. Correlações acima de 0,50 entre os fatores foram consideradas elevadas 42 .

Para o exame da consistência interna da CTSPC, foi calculado o coeficiente Kuder-Richardson-20. De acordo com Hair et al. ${ }^{29}$, um valor para o Kuder-Richardson superior a 0,70 já pode ser considerado satisfatório, sendo que valores superiores a 0,95 apontam para a possibilidade de ambiguidade entre os itens, visto que o grau de covariância entre os itens pode demonstrar a medida do mesmo atributo do construto 43 .

\section{Resultados}

\section{Análise fatorial exploratória}

A AFE revelou que alguns itens apresentaram elevadas cargas fatoriais nas suas respectivas escalas, enquanto outros itens se comportaram diferentemente da proposta original. Conforme mostra a Tabela 3, os itens A ("Explicou por que o que ele(a) estava fazendo estava errado?”), E 
Análise fatorial exploratória (AFE) e consistência interna da Parent-Child Conflit Tactics Scale (CTSPC).

\begin{tabular}{|c|c|c|c|c|c|c|}
\hline Item & Escala & & Fator 1 & Fator 2 & Fator 3 & $1-h^{2}$ \\
\hline A & DNV & Explicou à criança por que o que ele(a) estava fazendo estava errado? & $-0,1406$ & $-0,0435$ & 0,7033 & 0,5338 \\
\hline B & DNV & $\begin{array}{l}\text { Colocou de castigo do tipo: mandou-o(a) ficar em seu quarto ou em } \\
\text { qualquer outro lugar? }\end{array}$ & 0,0324 & 0,3477 & 0,3051 & 0,6960 \\
\hline E & DNV & $\begin{array}{l}\text { Deu a ele(a) outra coisa para fazer em vez daquilo que ele(a) estava fazendo } \\
\text { de errado? }\end{array}$ & $-0,0750$ & 0,1351 & 0,4153 & 0,7895 \\
\hline Q & DNV & Tirou as regalias dele(a) ou o(a) deixou sem sair de casa? & $-0,0226$ & 0,3678 & 0,4054 & 0,6123 \\
\hline $\mathrm{F}$ & $\mathrm{AP}$ & Falou alto, berrou ou gritou com a criança? & 0,2558 & 0,4568 & 0,1363 & 0,5117 \\
\hline $\mathrm{J}$ & AP & Xingou, praguejou, rogou praga contra a criança? & 0,6006 & 0,1086 & 0,0479 & 0,5326 \\
\hline $\mathrm{L}$ & AP & Ameaçou expulsá-lo(a) ou enxotá-lo(a) de casa? & 0,8287 & $-0,4089$ & 0,3470 & 0,4222 \\
\hline $\mathrm{N}$ & $\mathrm{AP}$ & Ameaçou dar um tapa, mas não deu? & 0,1149 & 0,4374 & 0,1166 & 0,6820 \\
\hline U & AP & Chamou de burro(a), estúpido(a), preguiçoso(a) ou coisa parecida? & 0,3125 & 0,2570 & 0,1119 & 0,6947 \\
\hline C & $\mathrm{PC}$ & Sacudiu a criança? & 0,4330 & 0,3451 & $-0,0873$ & 0,5407 \\
\hline $\mathrm{D}$ & $\mathrm{PC}$ & $\begin{array}{l}\text { Bateu no bumbum dele(a) com alguma coisa como um cinto, chinelo, } \\
\text { escova de cabelo, vara ou outro objeto duro? }\end{array}$ & 0,0960 & 0,7413 & $-0,1062$ & 0,4015 \\
\hline $\mathrm{H}$ & $\mathrm{PC}$ & Deu uma palmada no bumbum da criança? & $-0,1426$ & 0,8430 & $-0,0870$ & 0,4510 \\
\hline$P$ & $\mathrm{PC}$ & Deu um tapa na mão, no braço ou na perna da criança? & 0,0724 & 0,6424 & 0,1413 & 0,4410 \\
\hline $\mathrm{R}$ & PC & Deu beliscão na criança? & 0,4040 & 0,3882 & $-0,0243$ & 0,5069 \\
\hline $\mathrm{V}$ & $\mathrm{PC}$ & Deu um tapa/bofetada no rosto, na cabeça ou nas orelhas da criança? & 0,6660 & 0,1289 & 0,1015 & 0,3924 \\
\hline G & MF & Bateu com a mão fechada ou deu um chute com força nele(a)? & 0,7459 & 0,0798 & $-0,2907$ & 0,3775 \\
\hline ○ & MF & $\begin{array}{l}\text { Bateu em alguma parte do corpo dele(a) diferente do bumbum com } \\
\text { alguma coisa como um cinto, chinelo, escova de cabelo, vara ou outro } \\
\text { objeto duro? }\end{array}$ & 0,2296 & 0,3583 & 0,0320 & 0,7085 \\
\hline $\mathrm{T}$ & MF & Jogou a criança no chão? & 0,7556 & 0,0506 & 0,1050 & 0,3364 \\
\hline I & MFG & Agarrou pelo pescoço e o(a) sacudiu? & 0,7372 & 0,1026 & $-0,3248$ & 0,3612 \\
\hline K & MFG & $\begin{array}{l}\text { Bateu muito na criança, ou seja, bateu nele(a) sem parar, o máximo que } \\
\text { conseguiu? }\end{array}$ & 0,6814 & 0,0533 & $-0,0787$ & 0,5058 \\
\hline \multicolumn{3}{|c|}{ Autovalor } & 6,18 & 5,57 & 1,99 & \\
\hline \multicolumn{3}{|c|}{ Variância explicada total (\%) } & 0,65 & 0,58 & 0,21 & \\
\hline \multicolumn{3}{|c|}{ Kuder-Richardson-20 } & 0,63 & 0,59 & 0,42 & \\
\hline
\end{tabular}

AP: agressão psicológica; DNV: disciplina não-violenta; MF: maus-tratos; MFG: maus-tratos físicos; PC: punição corporal.

("Deu a ele(a) outra coisa para fazer em vez daquilo que ele(a) estava fazendo de errado?”) e Q ("Tirou as regalias dele(a) ou o(a) deixou sem sair de casa?”) da escala de disciplina não-violenta apresentaram cargas fatoriais altas no Fator 3. Porém, o item B ("Colocou de castigo do tipo: mandou-o(a) ficar em seu quarto ou em qualquer outro lugar?"), pertencente à mesma escala, apresentou carga fatorial mais alta no Fator 2, ainda que a diferença entre valores absolutos da carga fatorial apresentada no Fator 3 tivesse sido inferior a 0,10 .

Os itens J ("Xingou, praguejou, rogou praga contra a criança?”), L (“Ameaçou expulsálo(a) ou enxotá-lo(a) de casa?") e U (“Chamou de burro(a), estúpido(a), preguiçoso(a) ou coisa parecida?"), pertencentes à escala de agressão psicológica, apresentaram cargas fatoriais mais altas no Fator 1, enquanto que os demais itens dessa escala apresentaram cargas fatoriais mais altas no Fator 2.

Ao examinar os itens supostamente pertencentes à escala de punição corporal, observou-se que os itens D ("Bateu no bumbum dele(a) com alguma coisa como um cinto, chinelo, escova de cabelo, vara ou outro objeto duro?"), H ("Você deu uma palmada no bumbum da criança?") e P ("Deu um tapa/bofetada no rosto, na cabeça ou nas orelhas da criança?”) apresentaram cargas fatoriais mais altas no Fator 2, mas os demais itens tiveram cargas fatoriais mais altas no Fator 1, ainda que os itens C ("Sacudiu a criança?) e R (“Deu beliscão na criança?”), dessa mesma escala, tivessem atingido valores absolutos muito próximos nos Fatores 1 e 2, com diferença inferior a 0,10 . 
Já os itens pertencentes à escala de maus-tratos físicos tiveram cargas fatoriais mais altas no Fator 1, exceto o item O ("Bateu em alguma parte do corpo dele(a) diferente do bumbum com alguma coisa como um cinto, chinelo, escova de cabelo, vara ou outro objeto duro?") cuja carga maior alocou-se no Fator 2. Observou-se também proximidade de valores alcançados pelo item nos Fatores 1 e 2 com diferença inferior a 0,10. Os itens $M$ ("Queimou a criança ou derramou líquido quente nele(a) de propósito?”) e S (“Ameaçou com uma faca ou arma?") foram excluídos por terem apresentado baixa frequência de respostas.

O Fator 1 pareceu ter agregado itens com maior grau de violência por ter apresentado a maioria dos itens de maus-tratos físicos, o tipo de violência física considerado mais grave. Já o Fator 2 pareceu agregar itens que sugerem um grau médio de violência contra a criança, por ter englobado a maioria dos itens de punição corporal, o tipo de violência física considerado apenas disciplinadora. Tanto o Fator 1 quanto o Fator 2 apresentaram correlações com itens correspondentes à escala de agressão psicológica. O Fator 3 foi o que apresentou correlações mais baixas entre os demais itens da escala, podendo revelar táticas de resolução de conflito disciplinadoras não-violentas.

\section{Análise fatorial confirmatória}

A partir do agrupamento dos itens revelados na AFE, foi realizada a AFC. O Fator 1, denominado maus-tratos não-disciplinadores (MND), incluiu itens das escalas de maus-tratos físicos, punição corporal e agressão psicológica; o Fator 2, denominado maus-tratos disciplinadores (MTD), incluiu, em sua maioria, os itens de punição corporal e agressão psicológica e apenas um item de maus-tratos físicos; o Fator 3 foi nomeado como uso de táticas disciplinadoras não-violentas, o qual incluiu apenas os itens da escala de disciplina não-violenta (DNV) (Figura 1).

Os índices de adequação do modelo testado mostraram-se adequados (Tabela 4). O comparative fix index e o Tucker-Lewis index apresentaram estimativas acima de 0,90, indicando que o modelo encontra-se adequado.

Os parâmetros estimados foram todos significativos, e a correlação entre os fatores também foi alta. A correlação mais alta ocorreu entre os Fatores 3 e $2(0,802)$, e a mais baixa, entre os Fatores 1 e $3(0,469)$.

\section{Consistência interna}

Realizada a análise fatorial, examinou-se o nível de consistência interna da CTSPC para cada escala. Todos os valores obtidos para o KuderRichardson foram inferiores a 0,70 , sendo 0,63 para o Fator 1, 0,59 para o Fator 2 e 0,42 para o Fator 3. Não houve itens que, se retirados, melhorassem significativamente a consistência interna das escalas (Tabela 1).

\section{Discussão}

A AFC demonstrou a adequação do modelo, testado inicialmente por meio da análise fatorial exploratória, indicando que a validade de construto da CTSPC poderia ser revista. A AFE identificou que os itens analisados comportaram-se de forma diferente da proposta original do instrumento, demonstrando que há uma estrutura dimensional distinta, o que também foi observado em outros estudos internacionais 21,25. Essa estrutura fatorial distinta também tem sido verificada em estudos que identificaram a coocorrência entre os vários tipos de violência 17,30, ainda que essas pesquisas não tenham utilizado a AFC.

Esse achado é confirmado por estudo realizado nos Estados Unidos 21, o qual encontrou itens pertencentes a diferentes dimensões com elevadas cargas fatoriais em um mesmo fator, conduzindo à construção de novas dimensões ao considerar tais correlações. A gradação apresentada nos níveis de violência foi corroborada pelos achados da pesquisa realizada no Canadá 25 e sinalizaram similaridades com a tipologia proposta por Steinmetz 31 .

Alguns itens pareceram apresentar discrepâncias culturais relevantes que devem ser consideradas na interpretação dos dados. Isso pode ser observado no item V ("Deu um tapa/bofetada no rosto, na cabeça ou nas orelhas da criança?”), originalmente inserido na escala de punição corporal, e no item F ("Falou alto, berrou ou gritou com a criança?”), originalmente proposto na escala de agressão psicológica. Ambos apresentaram elevadas cargas fatoriais em fatores representantes de grau mais elevado de violência, comportamento similar foi observado em estudo no Rio de Janeiro 16, sugerindo uma estrutura dimensional diferente do estudo norte-americano onde o instrumento foi elaborado.

A AFE revelou também que alguns itens (U_AP, C_PC, R_PC e O_MF) apresentaram problemas relativos a cargas fatoriais similares em dois fatores, com diferença entre elas inferior a 0,10. O item B ("Colocou de castigo do tipo: mandou-o(a) ficar em seu quarto ou em qualquer outro lugar?”), pertencente à escala de disciplina não-violenta, teve carga fatorial mais elevada no Fator 2, correspondente a um grau moderado de violência. Esse achado, apesar de não demons- 
trar similaridade com o domínio teórico do construto, pode revelar uma possível associação entre o uso da negociação e a violência como disciplinamento. No entanto, após AFC, esse item se inseriu melhor no Fator 1, como na escala original, apresentando correlação alta com o construto disciplina não-violenta. Cargas similares em dois fatores e a não correspondência teórica entre o item e seu fator sugerem mais algumas considerações. É possível que tais achados indiquem imprecisões na forma como as perguntas foram elaboradas, ausência desses fenômenos na cultura brasileira ou aspectos de desejabilidade social. Sobre esse último, os respondentes podem ter fornecido informações consideradas mais aceitáveis ou aprovadas socialmente, negando a associação pessoal com opiniões e comportamentos que seriam reprováveis 44 . Por ser um tema tabu, muitos respondentes tendem a não revelar as táticas de conflito utilizadas com receio de denúncias ou represálias. Ainda que cuidados para evitar esses aspectos tenham sido tomados, é possível que a baixa frequência de respostas em alguns itens tenha ocasionando problemas no carregamento dos itens ou na sua distribuição entre os fatores. A retirada de itens da análise devido à baixa frequência de respostas parece ser procedimento comum, tendo em vista a ocorrência prévia em outros estudos, tanto no Brasil 16 como nos Estados Unidos 21 e no Canadá 15.

Figura 1

Resultado da análise fatorial confirmatória (AFC) para o modelo testado da Parent-Child Conflit Tactics Scale (CTSPC).

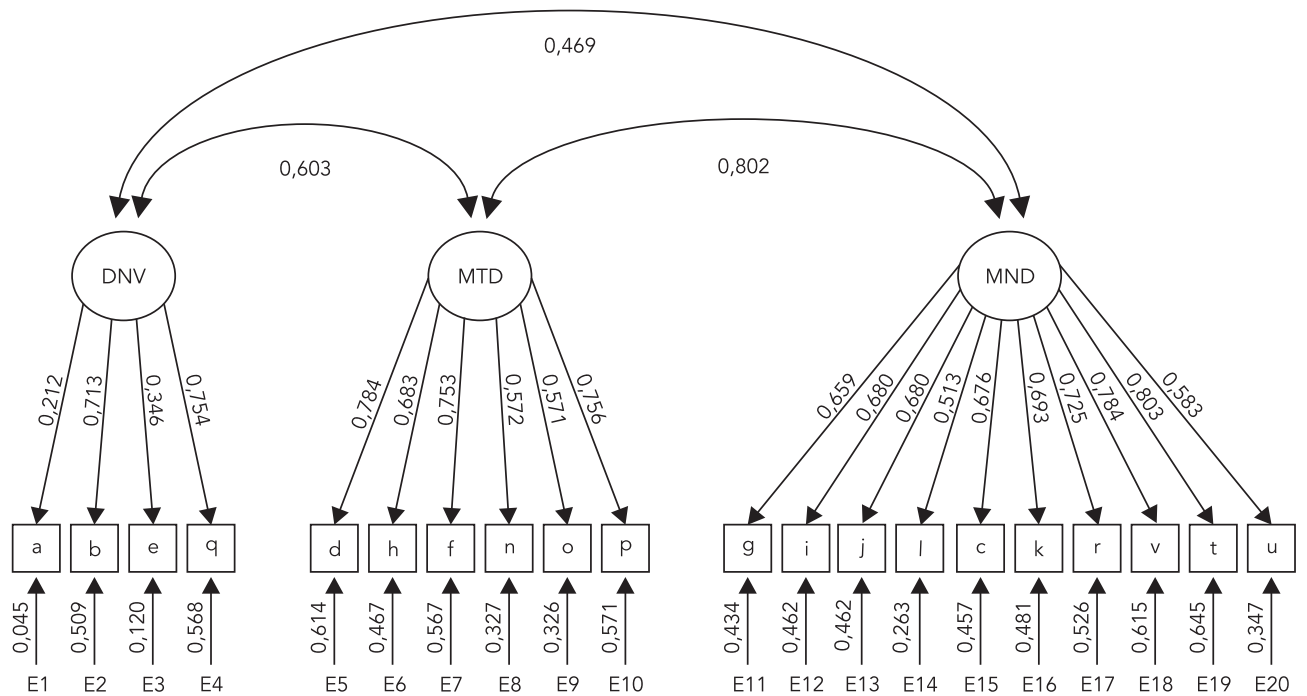

DNV: disciplina não-violenta; MND: maus-tratos não disciplinadores; MTD: maus-tratos disciplinadores.

Tabela 4

Índices de adequação do modelo testado da Parent-Child Conflit Tactics Scale (CTSPC) por meio de análise fatorial confirmatória (AFC).

\begin{tabular}{lcc}
\hline Índices & Estimativas & IC95\% \\
\hline Comparative fit index & 0,959 & - \\
Tucker-Lewis index & 0,954 & - \\
Root mean square error of approximation & 0,033 & $0,029-0,037$ \\
\hline
\end{tabular}

IC95\%: intervalo de 95\% de confiança. 
Outra diferença observada por este estudo refere-se ao fato de termos encontrado apenas três fatores, enquanto que a maioria dos estudos encontrou quatro fatores. Isto sugere que a estrutura dimensional mostra-se variável, conforme evidências de pesquisa canadense 25 que identificou apenas dois fatores.

As altas correlações identificadas na AFC podem indicar também que o construto violência doméstica contra a criança não poderia ser estudado de forma segmentada como propõe o instrumento originalmente. Analisando as correlações entre os fatores, a maior correlação ocorreu com os Fatores 2 e 3, os quais agregam itens referentes aos maus-tratos disciplinares e não-disciplinares. Esses achados demonstram o distanciamento entre esses fatores e o Fator 1, o qual agrega itens considerados como disciplinadores não-violentos.

A consistência interna mostrou-se similar aos valores encontrados nos demais estudos $17,22,23,24,25,28,30$, sendo o valor mais alto para o Fator 1 e o menor para o Fator 3. De forma similar aos estudos internacionais, o Fator 3, o qual agrega itens de MND, apresentou menor consistência interna, podendo indicar os problemas relativos à baixa frequência de respostas.

Baseando-se nessas evidências, é possível sugerir o redimensionamento da CTSPC, visto que a proposta original do instrumento não se mostra sustentável. A fragilidade da dimensionalidade da CTSPC pode ser detectada igualmente nos níveis de consistência interna das dimensões, visto que todas elas apresentaram valores para os coeficientes inferiores a 0,70 .

Embora tenham sido apontados problemas na dimensionalidade da escala, este estudo contribui para a compreensão da investigação do fenômeno da violência doméstica contra a criança. Além disso, o estudo avança no conhecimento com relação ao estudo de validação brasileiro 28 , pois realiza uma análise fatorial exploratória com rotação promax, utiliza o coeficiente Kuder-Richardson para avaliar a consistência interna da CTSPC e realiza a AFC, sugerindo um novo dimensionamento da escala.
Os instrumentos adaptados para a realidade brasileira são escassos, e os existentes necessitam ainda de modificações. Sendo um problema de saúde pública de alta magnitude na realidade brasileira, porém de tão difícil mensuração, a violência doméstica contra a criança é um tema que necessita de estudos mais aprofundados, com investigações mais acuradas para contribuição das estratégias de prevenção e promoção da saúde das famílias vitimizadas. A importância para a saúde pública torna-se premente, tendo em vista que atualmente a violência doméstica contra a criança tem sido identificada como fator de risco para doenças de prevalência elevada tais como asma, obesidade e síndrome do estresse póstraumático. A prevenção da violência doméstica contra crianças pode reduzir os custos para o setor de saúde e promover o bem-estar delas.

\section{Conclusões}

Este estudo colabora com a avaliação do construto violência doméstica contra a criança ao apresentar a análise da validade de construto da CTSPC a partir de um estudo de base populacional em um segundo contexto brasileiro. Buscou-se contribuir com a validade transcultural do instrumento, sendo identificadas características culturais similares ao estudo realizado no Rio de Janeiro. Uma vez investigada a validade de construto da CTSPC no contexto brasileiro, as diferenças encontradas entre este estudo e a proposta original do instrumento sugerem que o fenômeno violência doméstica contra a criança pode ser interpretado de formas distintas, tendo em vista a nova estrutura dimensional apresentada neste estudo. Corrobora também com o novo dimensionamento encontrado nos estudos canadense 19 e norte-americano 14 . Esses achados concorrentes dão indícios de que modificações futuras nos itens da CTSPC poderiam melhorar a qualidade psicométrica geral da medida. A relevância do tema e a necessidade de instrumentos que avaliem adequadamente o fenômeno ratificam a importância da investigação da estrutura do instrumento. 


\section{Resumo}

A Parent-Child Conflict Tactics Scale (CTSPC) tem sido um dos instrumentos mais utilizados internacionalmente para investigação da violência doméstica contra a criança, porém o uso segmentado de suas escalas tem se mostrado inadequado devido à complexidade do fenômeno. Esta pesquisa tem por objetivo estudar a estrutura fatorial das escalas da CTSPC em uma amostra populacional urbana do Nordeste brasileiro, sendo conduzido um estudo transversal com 1.370 crianças de uma coorte em Salvador, Bahia. Realizouse análise fatorial com rotação oblíqua promax e calculou-se o coeficiente Kuder-Richardson. A análise fatorial revelou uma distribuição dos itens nos fatores de forma diferente da proposta do instrumento original. Verificou-se um perfil gradativo de violência em cada fator. Os valores obtidos para o Kuder-Richardson foram 0,63 para o Fator 1, 0,59 para o Fator 2 e 0,42 para o Fator 3. Os itens comportaram-se de forma diferente da proposta original do instrumento, corroborando estudos internacionais. Baseando-se nessas evidências, é possivel propor um redimensionamento da CTSPC.

Maus-Tratos Infantis; Violência Doméstica; População Urbana; Análise Fatorial

\section{Colaboradores}

C. B. Bonfim contribuiu com a elaboração da pergunta do presente estudo, realizando revisão da bibliografia, análise dos dados e redação das diversas versões do artigo. D. N. Santos realizou a concepção do projeto e orientação de todas as etapas do estudo, contribuindo para a definição dos métodos de pesquisa, elaboração do modelo teórico, escrita e revisão do artigo. I. G. Menezes contribuiu para a análise do questionário sobre violência doméstica contra a criança, análise e interpretação dos dados, escrita e revisão do artigo. M. E. Reichenheim contribuiu na interpretação dos dados, escrita e revisão crítica do artigo. M. L. Barreto contribuiu na elaboração do desenho de estudo, interpretação dos dados, leitura e edição do manuscrito.

\section{Referências}

1. Douglas EM. Familial violence socialization in childhood and later life approval of corporal punishment: a cross-cultural perspective. Am J Orthopsychiatry 2006; 76:23-30.

2. Mascarenhas MDM, Malta DC, Silva MMA, Lima CM, Carvalho MGO, Oliveira VLA. Violência contra a criança: revelando o perfil dos atendimentos em serviços de emergência, Brasil, 2006 e 2007. Cad Saúde Pública 2010; 26:347-57.

3. Brasil. Lei $n^{\circ}$. 8.069. Dispõe sobre o Estatuto da Criança e do Adolescente e dá outras providências. Diário Oficial da União 1990; 16 jul.

4. Brasil. Portaria ${ }^{\circ}$. 737. Dispõe sobre a Política Nacional de Redução da Morbimortalidade por Acidentes e Violências. Diário Oficial da União 2001; 18 mai.
5. Brasil. Portaria no ${ }^{\circ}$ 936. Dispõe sobre a estruturação da Rede Nacional de Prevenção da Violência e Promoção da Saúde e a Implantação e Implementação de Núcleos de Prevenção à Violência em Estados e Municípios. Diário Oficial da União 2004; 20 mai.

6. Reichenheim ME, Hasselmann MH, Moraes CL. Consequências da violência familiar na saúde da criança e do adolescente: contribuições para a elaboração de propostas de ação. Ciênc Saúde Coletiva $1999 ; 1: 109-21$.

7. Pasquali L. Técnicas de exame psicológico - TEP São Paulo: Casa do Psicólogo; 2001. 
8. Duarte CS, Bordin IAS, Green GR, Hoven CW. Measuring child exposure to violence and mental health reactions in epidemiological studies: challenges and current issues. Ciênc Saúde Coletiva 2009; 14:487-96.

9. Briere J. Methodological issues in the study of sexual abuse effect. J Consult Clin Psychol 1992; 2:196-203.

10. Faleiros JM, Matias ASA, Bazon MR. Violência contra crianças na cidade de Ribeirão Preto, São Paulo, Brasil: a prevalência dos maus-tratos calculada com base em informações do setor educacional. Cad Saúde Pública 2009; 25:337-48.

11. Faleiros JM, Bazon MR. Estudo de prevalência de maus-tratos em crianças matriculadas de 1a a $4 \underline{a}$ série do ensino fundamental, nas escolas da rede pública e particular da cidade de Ribeirão Preto In: Livro de Resumos do 8o Seminário de Pesquisa. Ribeirão Preto: Legis Suma; 2005. p. 112.

12. Grassi-Oliveira R, Stein LM, Pezzi JC. Tradução e validação de conteúdo da versão em português do Childhood Trauma Questionnaire. Rev Saúde Pública 2006; 40:249-55.

13. Bernstein DP, Ahluvalia T, Pogge D, Handelsman L. Validity of the Childhood Trauma Questionnaire in an adolescent psychiatric population. J Am Acad Child Adolesc Psychiatry 1997; 36:340-8.

14. Bordin IAS, Paula CS, Nascimento R, Duarte CS. Severe physical punishment and mental health problems in an economically disadvantaged population of children and adolescents. Rev Bras Psiquiatr 2006; 28:290-6.

15. Sadowski LS, Hunter WM, Bangdiwala SI, Munoz SR. The World Studies of Abuse in the Family Environment (WorldSAFE): a model of a multi-national study of family violence. Int J Inj Contr Saf Promot 2004; 2:81-90.

16. Reichenheim ME, Moraes CL. Adaptação transcultural do instrumento Parent-Child Conflict Tactics Scales (CTSPC) utilizado para identificar a violência contra a criança. Cad Saúde Pública 2003; 6:1701-12.

17. Straus MA, Hamby SL, Finkelhor D, Moore DW, Runyan D. Identification of child maltreatment with the Parent-Child Conflict Tactics Scales: development and psychometric data for a national sample of American Parents. Child Abuse Negl 1998; 22:249-70

18. Leung PWS, Wong WCW, Chen WQ, Tang CSK. Prevalence and determinants of child maltreatment among high school students in Southern China: a large scale school based survey. Child Adolesc Psychiatry Ment Health 2008; 2:1-8.

19. Feigelman S, Dubowitz H, Lane W, Prescott L, Meyer W, Tracy K, et al. Screening for harsh punishment in a pediatric primary care clinic. Child Abuse Negl 2009; 33:269-77.

20. Duggan A, McFarlane E, Fuddy L, Burrell L, Higman SM, Windham A, et al. Randomized trial of a statewide home visiting program: impact in preventing child abuse and neglect. Child Abuse Negl 2004; 28:597-622.

21. Windham AM, Rosenberg L, Fuddy L, McFarlane E, Sai C, Duggan AK. Risk of mother-reported child abuse in the first 3 years of life. Child Abuse Negl 2004; 28:647-69.
22. Lee SJ, Guterman NB, Lee Y. Risk factors for paternal physical child abuse. Child Abuse Negl 2008; 32:846-58.

23. Downs WR, Capshew T, Rindels B. Relationships between adult women's mental health problems and their childhood experiences of parental violence and psychological aggression. J Fam Violence 2006; (21):439-47.

24. Dietz TL. Disciplining children: characteristics associated with the use of corporal punishment. Child Abuse Negl 2000; 24:1529-42.

25. Clement ME, Bouchard C. Predicting the use of single versus multiple types of violence towards children in a representative sample of Quebec families. Child Abuse Negl 2005; 29:1121-39.

26. Straus M. Translations of the CTSPC. http://pub pages.unh.edu/ mas2/CTS35C.pdf (acessado em 10/Abr/2009).

27. Mello MF, Schoedl AF, Pupo MC, Souza AAL, Andreoli SB, Bressan RA, et al. Adaptação transcultural e consistência interna do Early Trauma Inventory (ETI). Cad Saúde Pública 2010; 26:713-24.

28. Reichenheim ME, Moraes CL. Psychometric properties of the Portuguese version of the Conflict Tactics Scales: Parent-child version (CTSPC) used to identify child abuse. Cad Saúde Pública 2006; 22:505-15.

29. Hair J, Anderson RE, Tatham RL, Black WC. Análise multivariada de dados. Porto Alegre: Bookman; 2005.

30. Kelleher KJ, Hazen AL, Coben JH, Wang Y, McGeehan J, Kohl P, et al. Self-reported disciplinary practices among women in the child welfare system: association with domestic violence victimization. Child Abuse Negl 2008; 32:811-8.

31. Steinmetz SK. The use of force for resolving family conflict: the training ground for abuse. Fam Coord 1977; 26:19-26

32. Barreto ML, Cunha SS, Alcântara-Neves N, Carvalho LP, Cruz AA, Stein RT, et al. Risk factors and immunological pathways for asthma and other allergic diseases in children: background and methodology of a longitudinal study in a large urban center in Northeastern Brazil (Salvador-SCAALA study). BMC Pulm Med 2006; 6:1-10.

33. Divgi DR. Calculation of the tetrachoric correlation coefficient. Psychometrika 1979; 44:169-72.

34. Gorsuch RL. Factor analysis. New Jersey: Lawrence Erlbaum; 1983.

35. Smith GT, McCarthy DM. Methodological considerations in the refinement of clinical assessment instruments. Psychol Assess 1995; 7:300-8.

36. Tabachnick BG, Fidell LS. Using multivariate statistics. Boston: Pearson Education; 2007.

37. Stevens J. Applied multivariate statistics for the social sciences. Mahwah: Lawrence Erlbaum; 1996.

38. Pilati R, Abbad G. Análise fatorial confirmatória da escala de impacto do treinamento no trabalho. Psicol Teor Pesqui 2005; 21:43-51.

39. Antunes C, Fontaine AM. Percepção de apoio social na adolescência: análise fatorial confirmatória da escala Social Support Appraisals. Paidéia (Ribeirão Preto) 2005; 15:355-66.

40. Pereira C, Camino L, Costa JB. Análise fatorial confirmatória do Questionário de Valores Psicossociais - QVP24. Estud Psicol (Natal) 2004; 9:505-12. 
41. Amorim LDAF, Fiaccone RL, Santos CAST, Santos TN, Moraes LTLP, Oliveira NF, et al. Structural Equation Modeling in epidemiology. Cad Saúde Pública 2010; 26:2251-62.

42. Shah S, Novak S, Stapleton LM. Evaluation and comparison of models of metabolic syndrome using confirmatory analysis. Eur J Epidemiol 2006; 21:343-9.
43. Menezes IG. Escalas de Intenções Comportamentais de Comprometimento Organizacional (EICCO): concepção, desenvolvimento, validação e padronização [Dissertação de Mestrado]. Salvador: Universidade Federal da Bahia; 2006.

44. Anastasi A, Urbina S. Testagem psicológica. Porto Alegre: Editora Artes Médicas; 2000.

Recebido em 10/Jun/2011

Versão final reapresentada em 19/Mai/2011 Aprovado em 12/Jul/2011 